Bundesgesundheitsbl 2015 - 58:111-112

DOI 10.1007/s00103-015-2117-6

Online publiziert: 14. Januar 2015

(c) Springer-Verlag Berlin Heidelberg 2015

\author{
H. Tönnies ${ }^{1} \cdot$ L. Schaade ${ }^{2}$ \\ ${ }^{1}$ Geschäftsstelle Gendiagnostik-Kommission, Robert Koch-Institut, Berlin, Deutschland \\ ${ }^{2}$ Zentrum für Biologische Gefahren und spezielle Pathogenese; Vizepräsident, Robert Koch-Institut, \\ Berlin, Deutschland
}

\title{
Genetische Information und Prävention
}

ser Ausgabe beschäftigen, einen ((gestrichen, weil aktuell bereits im vorderen Teil des Satzes auftaucht)), beispielhaften Einblick in die Materie „Genetische Information und Prävention“.

Die genetischen Analysemethoden haben sich in der letzten Dekade in vielerlei Hinsicht rasant entwickelt. Heute kann ein menschliches Genom in wenigen Laborarbeitstagen vollständig sequenziert werden. Und dies zu einem Preis, der vor wenigen Jahren unvorstellbar gewesen wäre. H.-G. Klein und I. Rost geben aus Sicht der Labormedizin und Humangenetik im ersten Artikel „Moderne Genetische Analysemethoden. Grundlage für eine genetisch basierte Prävention" einen umfassenden aktuellen Überblick als Einstieg für den interessierten Leser.

Die Analyse genetischer Information unterliegt, wie alle medizinischen Leistungen, hohen Ansprüchen an die Korrektheit der ermittelten genetischen Daten. Wie von M. Stuhrmann-Spangenberg in seinem Artikel „Qualitätssicherung in der humangenetischen Diagnostik“ einleitend dargestellt, werden genetische Untersuchungen auf eine spezielle Fragestellung häufig nur einmal im Leben durchgeführt und haben zudem direkte Bedeutung nicht nur für das Individuum sondern häufig auch für dessen Familienangehörige.

Es gelten in Deutschland bezüglich der Qualitätssicherung humangenetischer Diagnostik verschiedene gesetzliche Regelungen, Richtlinien sowie fachgesellschaftsbasierte Leitlinien, die häufig jeweils aufeinander Bezug nehmen. Diese stellen im Sinne des einzelnen Patienten eine hochwertige interne und externe Qualitätssicherung sicher. Nicht zuletzt die freiwillige Akkreditierung medizini- scher Laboratorien, die humangenetische Diagnostik durchführen, sowie deren regelmäßige Überprüfung durch Re-Akkreditierung, stellen aus Sicht des Autoren einen höchst empfehlenswerten Beitrag zur langfristigen Qualitätssicherung dar.

Der Kenntnisstand über das menschliche Genom mit seinen ca. 20.000 Genen ist heute größer als je zuvor. Nichtsdestotrotz sind durch die reine Kenntnis der genomischen Sequenzen und deren Varianten weder deren medizinische Relevanz noch die zugrundeliegenden genetischen Erkrankungen selbst wirklich in Gänze „verstanden“. Um tiefere Erkenntnisse zur Plastizität des menschlichen Genoms und die Interaktionen von Genen und Genprodukten mit zusätzlichen exogenen Faktoren zu erlangen, sind noch große Anstrengungen vonnöten. Wichtige Grundlage für den Erkenntnisgewinn im klinisch-genetischen Bereich sind neben großen Fallzahlen ein hoher Informationsgehalt bezüglich jeder einzelnen verwendeten Probe (Genomik, Proteomik, Metabolomik, Transkriptomik etc.). Hier spielen Biodatenbanken, die Bioproben und Daten von bereits erkrankten Personen sammeln, eine ebenso wichtige Rolle wie Biodatenbanken mit Materialien von Personen, die prospektiv im Rahmen epidemiologischer und populations-bezogener Studien erfasst wurden, um Assoziationen genetischer Merkmale zu identifizieren, die (mit-)ursächlich für eine Krankheit sind, und deren Auftreten evtl. durch Präventionsmaßnahmen verhindert oder gemildert werden kann. M. Hummel und C. Rufenach geben in ihrem Artikel „Biomaterialbanken als Grundlage für die Entwicklung genetisch basierter Präventionskonzepte“ hier einen Über- 
blick aus der Sicht vernetzter biobankbasierter Forschung.

Verlässliche genetische Informationen zur Prädiktion von Erkrankungen zu generieren, setzt neben qualitätsgesichert hochwertigen analytischen Möglichkeiten ein hohes methodisch-statistisches Verständnis voraus. R. Foraita, M. Jäger und I. Pigeot vom Leibniz-Institut für Präventionsforschung und Epidemiologie (BIPS) widmen sich im vierten Grundlagenartikel dieses Bandes mit dem Titel „Methodisch-statistische Herausforderungen an die genombasierte Vorhersage von Erkrankungen" diesem sehr komplexen Thema, unter Berücksichtigung der Bedeutung genetischer Tests aus Sicht der Public-Health-Perspektive.

Als ein Beispiel für die Umsetzung des Konzeptes "Genetische Information und Prävention" im täglichen Leben kann heute sicher das Neugeborenenscreening gelten - eine diagnostische Untersuchung im Rahmen einer genetischen Reihenuntersuchung, die bei Nachweis des Vorliegens der Zielerkrankung eine erfolgreiche, frühe Intervention und Prävention erst möglich macht. Hierzu liegt der Artikel „Neugeborenenscreening: ein Paradebeispiel für effektive Sekundärprävention" von U. Nennstiel-Ratzel, A. Lüders und $\mathrm{O}$. Blankenstein vor, der auch deutlich darstellt, wie wichtig die ständige Evaluation des Gesamtprozesses unter Qualitätsaspekten ist.

Die fachgerechte Nutzung genetischer Information zur Prävention setzt, wie die vorausgegangenen Artikel gezeigt haben, ein hohes Maß an Interdisziplinarität voraus. Der Artikel „Genetik und Prävention am Beispiel genetischer Aortensyndrome (GAS) und des Marfan Syndroms" spiegelt dies nochmals deutlich wider. Hier stellt ein interdisziplinäres Autorenteam der Fachdisziplinen Kardiologie, Gesundheitsökonomie, Herzchirurgie und Molekulargenetik zusammen mit einem Patientenverteter exemplarisch die wichtigsten Aspekte zur Genetik und Prävention an den Beispielen der GAS und des Marfan Syndroms eindrücklich dar.

Vor der eigentlichen Etablierung möglicher Präventionskonzepte im Zusammenhang mit genetisch determinierten komplexen Erkrankungen in der klini- schen Praxis sind umfassende valide genetische Informationen unerlässlich. Dass bei komplexen klinischen Erkrankungen auch sogenannte epigenetische Informationen zum Teil eine erhebliche Rolle spielen, war vor gerade einmal einem Jahrzehnt nahezu unbekannt. Bei häufigen medizinischen Problemen wie Adipositas spielen neben einer Vielzahl von krankheitsassoziierten genetischen Varianten vor allem auch umweltbedingte Einflüsse eine wesentliche Rolle. Dieses komplexe Zusammenspiel von genetischen und epigenetischen Regulationsmechanismen genauer zu verstehen ist ein wesentlicher Aspekt der Adipositasforschung auf genetischer Ebene. A. Hinney, N. Herrfurth, L. Schonnop und A.-L. Volckmar beleuchten in ihrem Artikel "Genetik und Epigenetik der Adipositas" den aktuellen Stand der Erkenntnisse auf diesem Gebiet.

Doch nicht nur das Genom des Menschen selbst kann für bestehende und sich entwickelnde Erkrankungen des Individuums verantwortlich sein. Es gibt erste Hinweise darauf, dass auch die intestinalen Mikroorganismen und ihre Gene (Metagenom) mit dem Wirt in wechselseitige Interaktion treten. Wie genau das Metagenom die Gesundheit des Wirts erhalten oder auch schädigen kann, ist Fokus aktueller Untersuchungen. Dieses relativ junge und außerordentlich spannende Forschungsgebiet stellen D. Haller und G. Hörmannsperger in ihrem Artikel „Die Interaktion zwischen Darmbakterien und Mensch als zentraler Faktor der Darmgesundheit. Mikrobiom und chronisch entzündliche Darmerkrankungen“ dar.

Neben den o. g. Artikeln dieses Sonderbandes finden Sie zudem einen aktuellen Übersichtsartikel zur Mitteilung genetischer Zusatzbefunde aus der Geschäftsstelle der Gendiagnostikkommission beim RKI, den wir Ihnen natürlich ebenfalls gern empfehlen.

Liebe Leserinnen und Leser, wir wünschen Ihnen eine spannende und erkenntnisreiche Lektüre,

Ihre

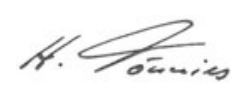

H. Tönnies

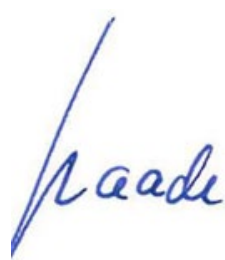

L. Schaade

\begin{tabular}{|l}
\hline Korrespondenzadressen \\
\hline $\begin{array}{l}\text { PD Dr. H. Tönnies } \\
\text { Geschäftsstelle Gendiagnostik- } \\
\text { Kommission } \\
\text { Robert Koch-Institut } \\
\text { Nordufer 20, 13353 Berlin } \\
\text { toenniesh@rki.de }\end{array}$ \\
\hline $\begin{array}{l}\text { PD Dr. L. Schaade } \\
\text { Zentrum für Biologische } \\
\text { Gefahren und spezielle } \\
\text { Pathogenese; Vizepräsident } \\
\text { Robert Koch-Institut } \\
\text { Nordufer 20, 13353 Berlin } \\
\text { schaadel@rki.de }\end{array}$ \\
\hline
\end{tabular}

